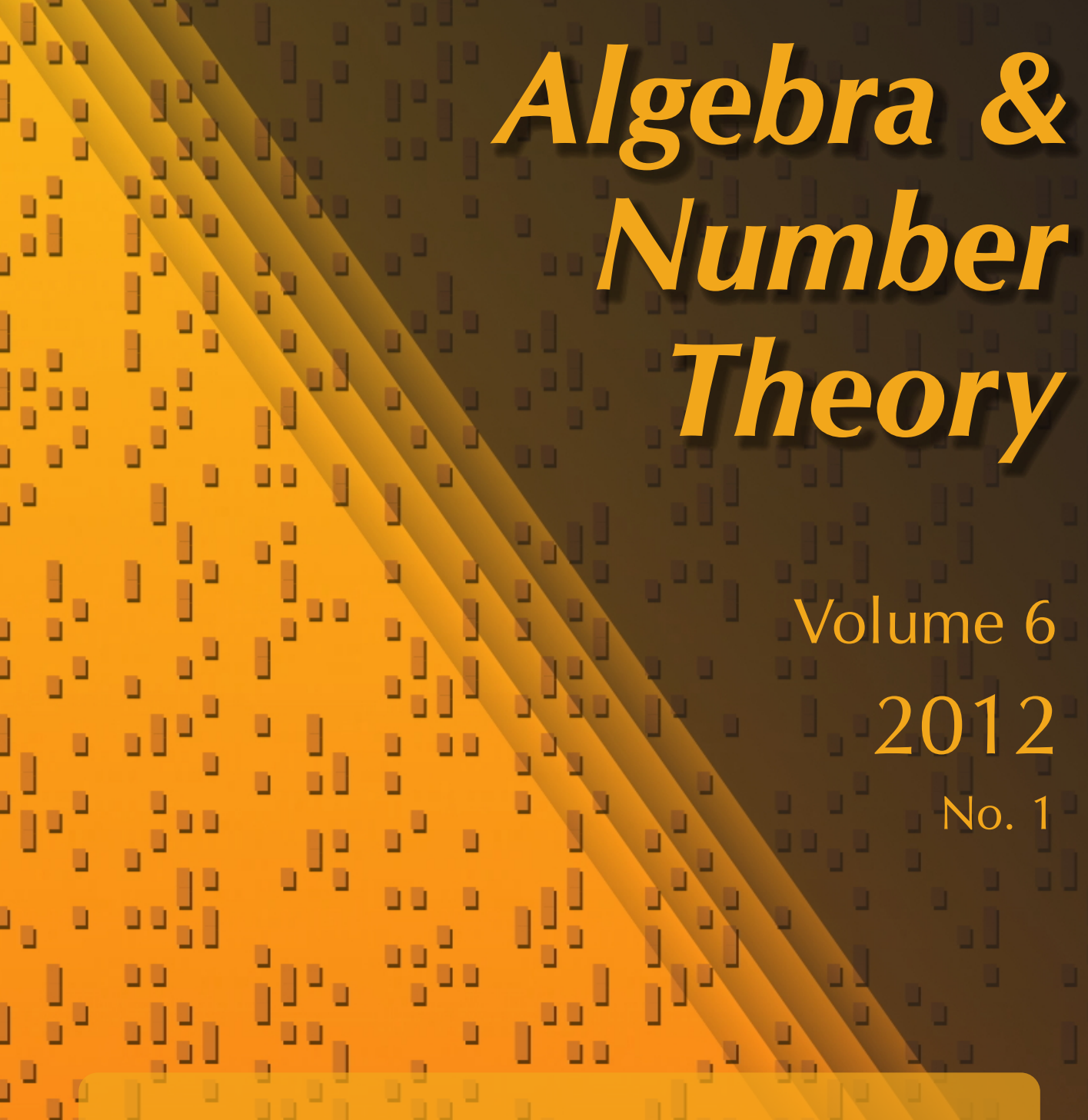

The minimal resolution conjecture for points on del Pezzo surfaces

\lrcorner$\lrcorner \quad$ Rosa M. Miró-Roig and Joan Pons-Llopis

」

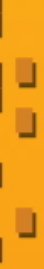




\title{
The minimal resolution conjecture for points on del Pezzo surfaces
}

\author{
Rosa M. Miró-Roig and Joan Pons-Llopis
}

Mustaţă (1997) stated a generalized version of the minimal resolution conjecture for a set $Z$ of general points in arbitrary projective varieties and he predicted the graded Betti numbers of the minimal free resolution of $I_{Z}$. In this paper, we address this conjecture and we prove that it holds for a general set $Z$ of points on any (not necessarily normal) del Pezzo surface $X \subseteq \mathbb{P}^{d}$ - up to three sporadic cases - whose cardinality $|Z|$ sits into the interval $\left[P_{X}(r-1), m(r)\right]$ or $\left[n(r), P_{X}(r)\right], r \geq 4$, where $P_{X}(r)$ is the Hilbert polynomial of $X, m(r):=$ $\frac{1}{2} d r^{2}+\frac{1}{2} r(2-d)$ and $n(r):=\frac{1}{2} d r^{2}+\frac{1}{2} r(d-2)$. As a corollary we prove: (1) Mustaţă's conjecture for a general set of $s \geq 19$ points on any integral cubic surface in $\mathbb{P}^{3}$; and (2) the ideal generation conjecture and the Cohen-Macaulay type conjecture for a general set of cardinality $s \geq 6 d+1$ on a del Pezzo surface $X \subseteq \mathbb{P}^{d}$.

\section{Introduction}

Given a general set $Z$ of $s$ distinct points in $\mathbb{P}^{n}$ it is a long-standing problem in algebraic geometry to find out the exact shape of the minimal free resolution of its saturated ideal $I_{Z}$. It is well-known that it has to be of the form

$$
0 \rightarrow F_{n} \rightarrow \cdots \rightarrow F_{1} \rightarrow I_{Z} \rightarrow 0
$$

with

$$
F_{i} \cong R(-r-i)^{b_{i, r}} \oplus R(-r-i+1)^{b_{i, r-1}},
$$

where $R$ is the coordinate ring of $\mathbb{P}^{n}$ and $r$ is the unique nonnegative integer such that $\left(\begin{array}{c}r+n-1 \\ n\end{array}\right) \leq s<\left(\begin{array}{c}r+n \\ n\end{array}\right)$. Moreover,

$$
b_{i+1, r-1}-b_{i, r}=\left(\begin{array}{c}
r+i-1 \\
i
\end{array}\right)\left(\begin{array}{c}
r+n \\
n-i
\end{array}\right)-s\left(\begin{array}{l}
n \\
i
\end{array}\right) .
$$

The first author was partially supported by MTM2010-15256. The second author was supported by the research project MTM2009-06964.

MSC2000: primary 13D02; secondary 13D40, 14M05.

Keywords: minimal free resolutions, del Pezzo surfaces, $G$-liaison. 
The minimal resolution conjecture (MRC, for short) stated in [Lorenzini 1993] says that this resolution has no ghost terms, that is, $b_{i+1, r-1} b_{i, r}=0$ for all $i$. The MRC is known to hold for $n \leq 4$ [Gaeta 1951; Ballico and Geramita 1986; Walter 1995] and for large values of $s$ for any $n$ [Hirschowitz and Simpson 1996] but it is false in general: Eisenbud, Popescu, Schreyer and Walter showed that it fails for any $n \geq 6, n \neq 9$ (see [Eisenbud et al. 2002]).

Besides MRC, two weaker conjectures have been stated concerning the initial and ending terms of the minimal free resolution of an ideal of points: the ideal generation conjecture (IGC for short), which says that the minimal number of generators of the ideal of a general set of points will be as small as possible; this conjecture can be translated in terms of the Betti numbers saying that $b_{1, r} b_{2, r-1}=0$. At the other extreme of the resolution the Cohen-Macaulay type conjecture (CMC for short) affirms that the canonical module $\operatorname{Ext}_{R}^{n}\left(R / I_{Z}, R(-n-1)\right)$ has as few generators as possible, i.e., $b_{n-1, r} b_{n, r-1}=0$.

Mustață [1998] introduced a generalized version of MRC for points in arbitrary projective varieties (see Section 2 for a precise statement). Roughly speaking, it says that given a projective variety $X \subseteq \mathbb{P}^{n}$, the minimal free resolution of the ideal of any general set of points on $X$ is determined by the resolution of the ideal of $X$. When $X=\mathbb{P}^{n}$, this formulation coincides with the original Lorenzini's statement. Giuffrida, Maggioni and Ragusa proved that this generalized conjecture holds for any general set of points when $X$ is a smooth quadric surface in $\mathbb{P}^{3}$ [Giuffrida et al. 1996]. Casanellas [2009] proved that this conjecture holds for some special cardinalities of sets of general points on a smooth cubic surface. In [Miró-Roig and Pons-Llopis 2012] we showed that it also holds for any general set of at least 19 points on a smooth cubic surface in $\mathbb{P}^{3}$; Migliore and Patnott have been able to prove this for sets of general distinct points of any cardinality on a cubic surface $X \subseteq \mathbb{P}^{3}$ given that $X$ is smooth or has at most isolated double points [Migliore and Patnott 2011, Theorem 1]. For the case of nonreduced 0-dimensional schemes see [Miró-Roig and Pons-Llopis 2012].

The goal of this paper it to prove MRC for general points on a del Pezzo surface $\left(X, O_{X}(1)\right)$, i.e., an integral arithmetically Gorenstein (not necessarily normal) surface with a very ample line bundle $O_{X}(1)$ such that its dualizing sheaf verifies $\omega_{X} \cong O_{X}(-1)$. This kind of variety has been studied thoroughly by Fujita [1990] in connection with his theory of $\Delta$-genus. He defines the $\Delta$-genus of a polarized variety $\left(X, \mathscr{O}_{X}(1)\right)$ of dimension $n$ as $\Delta\left(X, \mathscr{O}_{X}(1)\right):=n+\mathscr{O}_{X}(1)^{n}-\mathrm{h}^{0}\left(X, \mathscr{O}_{X}(1)\right)$. In his terminology, del Pezzo varieties are ACM varieties of $\Delta$-genus one.

The main technique used in this paper is the theory of Gorenstein liaison (see Section 2 for a brief account). Roughly speaking, knowing that two sets of points are $G$-linked will allow to pass from the minimal resolution of the ideal of one of them to the resolution of the other one (mapping cone procedure). Then once MRC 
is known to hold for a general set of $d+2$ points on a del Pezzo surface $X \subseteq \mathbb{P}^{d}$ an induction process will provide us with our main theorems (4.2, 4.3 and 4.4).

Let us briefly explain how this paper is organized. In Section 2 we introduce the background and main techniques needed, including general facts on del Pezzo surfaces and basic results on G-liaison. In Section 3 we establish MRC for sets of general points of two specific cardinalities,

$$
m(r):=\frac{1}{2} d r^{2}+\frac{1}{2} r(2-d) \text { and } n(r):=\frac{1}{2} d r^{2}+\frac{1}{2} r(d-2),
$$

with $r \geq 2$, on a del Pezzo surface $X \subset \mathbb{P}^{d}$ (up to four sporadic cases). We first establish the result for $m(2)$ points on $X$, which gives the initial step of our induction (Lemma 3.5). An easy remark gives us that if $n(r)$ general points on $X$ have the expected resolution then $n(r)+1$ general points do as well. Then, using G-liaison, we prove that if $m(r)$ general points on a del Pezzo surface $X$ satisfy MRC then so do $n(r)$ general points (Proposition 3.6). Finally, again using Gliaison, we show that if $n(r)+1$ general points on a del Pezzo surface satisfy MRC then so do $m(r+1)$ (Proposition 3.8). Section 4 contains the main results of this paper: namely that MRC holds on a del Pezzo surface (up to three of the four sporadic cases just mentioned) for general sets of points whose cardinality falls in the intervals $\left[P_{X}(r-1), m(r)\right]$ and $\left[n(r), P_{X}(r)\right]$ for any $r \geq 4=\operatorname{reg} X+1$, with $P_{X}(r)$ the Hilbert polynomial (see Theorem 4.2). As a corollary, we will get that Mustață's conjecture holds for any general set of at least 19 points on a cubic surface in $\mathbb{P}^{3}$ (Theorem 4.4 ) and the ideal generation conjecture as well as the Cohen-Macaulay type conjecture holds for any general set of at least $6 d+1$ points on a del Pezzo surface in $\mathbb{P}^{d}$ (Theorem 4.3).

\section{Preliminaries}

We work over an algebraically closed field $k$ of characteristic zero. We set $R=$ $k\left[X_{0}, \ldots, X_{n}\right]$ and denote the associated projective space by $\mathbb{P}^{n}:=\operatorname{Proj}(R)$. Given closed subschemes $Y \subseteq X \subseteq \mathbb{P}^{n}$, we denote the ideal sheaf of $Y$ in $X$ by $\mathscr{I}_{Y \mid X}$ and the homogeneous saturated ideal by $I_{Y \mid X}:=\mathrm{H}_{*}^{0}\left(X, \mathscr{I}_{Y \mid X}\right)$ (or simply $I_{Y}$ when $\left.X=\mathbb{P}^{n}\right)$. We denote by $R_{X}$ the homogeneous coordinate ring of $X$, defined as $k\left[X_{0}, \ldots, X_{n}\right] / I_{X}$. For any coherent sheaf $\mathscr{E}$ on $X$ we denote the twisted sheaf $\mathscr{E} \otimes \mathscr{O}_{X}(l)$ by $\mathscr{E}(l)$. As usual, $\mathrm{H}^{i}(X, \mathscr{E})$ stands for the $i$-th cohomology group and $\mathrm{h}^{i}(X, \mathscr{E})$ for its dimension. We use the notation $\mathrm{H}_{*}^{i}(\mathscr{E})$ for the graded $R$-module $\bigoplus_{l \in \mathbb{Z}} \mathrm{H}^{i}\left(\mathbb{P}_{k}^{n}, \mathscr{E}(l)\right)$ and $\omega_{X}$ will stand for the dualizing sheaf. The Hilbert function and Hilbert polynomial of $X$ are denoted, respectively, by $H_{X}(t)$ and $P_{X}(t) \in \mathbb{Q}[t]$. The regularity of $X$ is defined to be that of $I_{X}$; i.e., reg $X \leq m$ if and only if $\mathrm{H}^{i}\left(\mathbb{P}^{n}, \mathscr{I}_{X}(m-i)\right)=0$ for $i \geq 1$. By [Eisenbud 2005, Chapter IV, Theorem 4.2] we know that $P_{X}(t)=H_{X}(t)$ for any $t \geq \operatorname{reg} X-1+\delta-n$, where $\delta$ is the projective dimension of $R_{X}$. By a variety we mean an integral and proper scheme over $k$. 
Definition 2.1. Let $X \subseteq \mathbb{P}^{n}$ be a subscheme with minimal graded free resolution

$$
F_{.}: 0 \rightarrow F_{n+1} \stackrel{d_{n+1}}{\longrightarrow} F_{n} \rightarrow \cdots \rightarrow F_{1} \stackrel{d_{1}}{\longrightarrow} F_{0} \stackrel{d_{0}}{\longrightarrow} R / I_{X} \rightarrow 0 .
$$

The graded Betti numbers $b_{i j}(X)$ are defined by

$$
F_{i}=\bigoplus_{j \in \mathbb{Z}} R(-i-j)^{b_{i j}(X)}, \quad \text { i.e., } \quad b_{i j}(X)=\operatorname{dim}_{k} \operatorname{Tor}^{i}\left(R / I_{X}, k\right)_{i+j}
$$

and the Betti diagram of $X$ has in the $(i, j)$-th position the Betti number $b_{i j}(X)$.

Remark 2.2. The free resolution $F_{\text {. }}$ is minimal if, after choosing basis of $F_{i}$, the matrices representing $d_{i}$ do not have any nonzero scalar.

Mustață [1998] predicted the minimal free resolution of a general set of points $Z$ in an arbitrary projective variety $X$; he proved that the first rows of the Betti diagram of $Z$ coincide with the Betti diagram of $X$ and that there are two extra nontrivial rows at the bottom. He also gave lower bounds for the Betti numbers in these last two rows and the minimal resolution conjecture (MRC) for points on a projective variety states that these lower bounds are attained for a general set of points. Let us recall it.

Theorem 2.3 [Mustață 1998]. Let $X \subseteq \mathbb{P}^{n}$ be a projective variety with $\operatorname{dim} X \geq 1$ and $\operatorname{reg} X=m$. Let $s$ be an integer with $P_{X}(r-1) \leq s<P_{X}(r)$ for some $r \geq m+1$ and let $Z$ be a set of $s$ general points on $X$. If

$$
0 \rightarrow F_{n} \rightarrow F_{n-1} \rightarrow \cdots \rightarrow F_{2} \rightarrow F_{1} \rightarrow R \rightarrow R / I_{X} \rightarrow 0
$$

is a minimal free $R$-resolution of $R / I_{X}$, then $R / I_{Z}$ has a minimal free $R$-resolution of the type

$$
\begin{aligned}
& 0 \rightarrow F_{n} \oplus R(-r-n+1)^{b_{n, r-1}} \oplus R(-r-n)^{b_{n, r}} \\
& \rightarrow \cdots \rightarrow F_{2} \oplus R(-r-1)^{b_{2, r-1}} \oplus R(-r-2)^{b_{2, r}} \\
& \rightarrow F_{1} \oplus R(-r)^{b_{1, r-1}} \oplus R(-r-1)^{b_{1, r}} \rightarrow R \rightarrow R / I_{Z} \rightarrow 0 ;
\end{aligned}
$$

moreover,

$$
b_{i+1, r-1}(Z)-b_{i, r}(Z)=\sum_{l=0}^{\operatorname{dim} X-1}(-1)^{l}\left(\begin{array}{c}
n-l-1 \\
i-l
\end{array}\right) \Delta^{l+1} P_{X}(r+l)-\left(\begin{array}{c}
n \\
i
\end{array}\right)\left(s-P_{X}(r-1)\right) \text {. }
$$

The minimal resolution conjecture (MRC for short) says that $b_{i+1, r-1} \cdot b_{i, r}=0$ for $i=1, \ldots, n-1$. Related to it are two weaker conjectures that deal only with a part of the minimal resolution of a general set of points: the ideal generation conjecture (IGC for short), which says that the minimal number of generators of the ideal of a general set of points will be as small as possible; this conjecture can be translated in terms of the Betti numbers saying that $b_{1, r} b_{2, r-1}=0$. At the other extreme of the resolution the Cohen-Macaulay type conjecture (CMC for short) 
affirms that the canonical module $\operatorname{Ext}_{R}^{n}\left(R / I_{Z}, R(-n-1)\right)$ has as few generators as possible, i.e., $b_{n-1, r} b_{n, r-1}=0$.

One of the main tools used in this paper is Gorenstein liaison theory. We recall its main features, of this theory referring the reader to [Kleppe et al. 2001] for a complete account.

Definition 2.4. A closed subscheme $X \subseteq \mathbb{P}^{n}$ of dimension $r$ is said to be Arithmetically Cohen-Macaulay (briefly, $A C M$ ) if its homogeneous coordinate ring $R_{X}$ is a Cohen-Macaulay ring or, equivalently, $\operatorname{dim} R_{X}=\operatorname{depth} R_{X}$.

Thanks to the graded version of the Auslander-Buchsbaum formula (for any finitely generated $R$-module $M$ ):

$$
\operatorname{pd}(M)=n+1-\operatorname{depth}(M),
$$

we deduce that a subscheme $X \subseteq \mathbb{P}^{n}$ is ACM if and only if the projective dimension of $R_{X}$ is equal to the codimension of $X$; i.e.,

$$
\operatorname{pd}\left(R_{X}\right)=\operatorname{codim} X .
$$

Hence, if $X \subseteq \mathbb{P}^{n}$ is a codimension $c$ ACM subscheme, a graded minimal free $R$-resolution of $I_{X}$ is of the form:

$$
0 \rightarrow F_{c} \rightarrow F_{c-1} \rightarrow \cdots \rightarrow F_{1} \rightarrow I_{X} \rightarrow 0
$$

where $F_{i}=\bigoplus_{j \in \mathbb{Z}} R(-j)^{\beta_{i, j}}, i=1, \ldots, c$.

Definition 2.5. If $X \subseteq \mathbb{P}^{n}$ is an ACM subscheme then, the rank of the last free $R$ module in a minimal free $R$-resolution of $I_{X}$ is called the Cohen-Macaulay type of $X$.

Definition 2.6. A codimension $c$ subscheme $X$ of $\mathbb{P}^{n}$ is arithmetically Gorenstein (briefly AG) if its homogeneous coordinate ring $R_{X}$ is a Gorenstein ring or, equivalently, its saturated homogeneous ideal, $I_{X}$, has a minimal free graded $R$-resolution of the following type:

$$
0 \rightarrow R(-t) \rightarrow \bigoplus_{i=1}^{\alpha_{c-1}} R\left(-n_{c-1, i}\right) \rightarrow \cdots \rightarrow \bigoplus_{i=1}^{\alpha_{1}} R\left(-n_{1, i}\right) \rightarrow I_{X} \rightarrow 0
$$

In other words, an AG scheme is an ACM scheme with Cohen-Macaulay type 1.

Any zero-dimensional scheme is ACM. For varieties of higher dimension we have the following characterization:

Lemma 2.7. If $\operatorname{dim} X \geq 1$, then $X \subseteq \mathbb{P}^{n}$ is $A C M$ if and only if $\mathrm{H}_{*}^{i}\left(\mathscr{I}_{X}\right)=0$ for $1 \leq i \leq \operatorname{dim} X$.

The following remark will be used without further mention throughout the paper: 
Remark 2.8. Let $X \subseteq \mathbb{P}^{n}$ be an ACM variety of dimension $\geq 1$ and let $Y \subseteq X$ be any subvariety. Then the saturated ideal $I_{Y \mid X}$ equals $I_{Y \mid \mathbb{P}^{n}} / I_{X \mid \mathbb{P}^{n}}$.

Definition 2.9. Two subschemes $X_{1}$ and $X_{2}$ of $\mathbb{P}^{n}$ are directly Gorenstein linked (directly G-linked for short) by an AG scheme $G \subseteq \mathbb{P}^{n}$ if $I_{G} \subseteq I_{X_{1}} \cap I_{X_{2}}$ and

$$
\left[I_{G}: I_{X_{1}}\right]=I_{X_{2}}, \quad\left[I_{G}: I_{X_{2}}\right]=I_{X_{1}} .
$$

We say that $X_{2}$ is residual to $X_{1}$ in $G$. When $G$ is a complete intersection we talk about a $C I$-link.

When $X_{1}$ and $X_{2}$ do not share any component, being directly $G$-linked by an AG scheme $G$ is equivalent to $G=X_{1} \cup X_{2}$.

Usually it is not easy to find out AG schemes to work with. The following theorem gives a useful way to construct them. Notice that, since we will want to work with varieties that can even be nonnormal, we will have to work in the framework of generalized divisors as introduced in [Hartshorne 1994; 2007]. The only general requirements to be fulfilled in order to work in this context are that the schemes satisfy conditions $S_{2}$ and $G_{1}$.

Definition 2.10. A subscheme $X \subseteq \mathbb{P}^{n}$ satisfies the condition $G_{r}$ if every localization of $R_{X}$ of dimension $\leq r$ is a Gorenstein ring. Usually this property is quoted as "Gorenstein in codimension $\leq r$ ", i.e., the non locally Gorenstein locus has codimension $\geq r+1$. In particular, $G_{0}$ is generically Gorenstein.

Theorem 2.11 (compare [Kleppe et al. 2001, Lemma 5.4]). Let $S \subseteq \mathbb{P}^{n}$ be an ACM scheme satisfying condition $G_{1}$. Denote by $K_{S}$ the canonical divisor and by $H_{S} a$ general hyperplane section of $S$. Then any effective divisor in the linear system $\left|m H_{S}-K_{S}\right|$ is arithmetically Gorenstein.

The main feature of $G$-liaison exploited in this paper is that through the mapping cone procedure it is possible to pass from the free resolution of a scheme $X_{1}$ to the free resolution of its residual $X_{2}$ on an arithmetically Gorenstein scheme. Let us recall how it works [Weibel 1994]:

Lemma 2.12 (mapping cone procedure). Let

$$
0 \rightarrow M \stackrel{\alpha}{\longrightarrow} N \rightarrow P \rightarrow 0
$$

be a short exact sequence of finitely generated $R$-modules and let us consider free resolutions

$$
e_{.}: 0 \rightarrow G_{n+1} \stackrel{e_{n+1}}{\longrightarrow} G_{n} \rightarrow \cdots \stackrel{e_{1}}{\longrightarrow} G_{0} \stackrel{e_{0}}{\longrightarrow} M \rightarrow 0
$$

and

$$
d_{\bullet}: 0 \rightarrow F_{n+1} \stackrel{d_{n+1}}{\longrightarrow} F_{n} \rightarrow \cdots \stackrel{d_{1}}{\longrightarrow} F_{0} \stackrel{d_{0}}{\longrightarrow} N \rightarrow 0
$$


Then the morphism $\alpha$ lifts to a morphism between the resolutions $\alpha_{\bullet}: e_{\bullet} \rightarrow d$. and a (not necessarily minimal) free resolution for $P$ is

$$
0 \rightarrow G_{n+1} \stackrel{c_{n+2}}{\rightarrow} G_{n} \oplus F_{n+1} \stackrel{c_{n+1}}{\rightarrow} \cdots \stackrel{c_{3}}{\rightarrow} G_{1} \oplus F_{2} \stackrel{c_{2}}{\rightarrow} G_{0} \oplus F_{1} \stackrel{c_{1}}{\rightarrow} F_{0} \stackrel{c_{0}}{\rightarrow} P \rightarrow 0,
$$

where

$$
c_{i+1}=\left(\begin{array}{cc}
-e_{i} & 0 \\
\alpha_{i} & d_{i+1}
\end{array}\right) \quad \text { for } 1 \leq i \leq n .
$$

Lemma 2.13. Let $V_{1}, V_{2} \subseteq \mathbb{P}^{n}$ be two $A C M$ schemes of codimension c directly $G$-linked by an $A G$ scheme $W$. Let the minimal free resolutions of $I_{V_{1}}$ and $I_{W}$ be

$$
0 \rightarrow F_{c} \stackrel{d_{c}}{\longrightarrow} F_{c-1} \stackrel{d_{c-1}}{\longrightarrow} \cdots F_{1} \stackrel{d_{1}}{\longrightarrow} I_{V_{1}} \rightarrow 0
$$

and

$$
0 \rightarrow R(-t) \stackrel{e_{c}}{\longrightarrow} G_{c-1} \stackrel{e_{c-1}}{\longrightarrow} \cdots G_{1} \stackrel{e_{1}}{\longrightarrow} I_{W} \rightarrow 0
$$

respectively. Then the functor $\operatorname{Hom}(-, R(-t))$ applied to a free resolution of $I_{V_{1}} / I_{W}$ gives a (not necessarily minimal) resolution of $I_{V_{2}}$ :

$$
0 \rightarrow F_{1}^{\vee}(-t) \rightarrow F_{2}^{\vee}(-t) \oplus G_{1}^{\vee}(-t) \rightarrow \cdots \rightarrow F_{c}^{\vee}(-t) \oplus G_{c-1}^{\vee}(-t) \rightarrow I_{V_{2}} \rightarrow 0 .
$$

Let us finish this section introducing the family of varieties that we deal with throughout this paper.

Definition 2.14. A del Pezzo surface is a nondegenerate 2-dimensional projective variety $X \subseteq \mathbb{P}^{d}$ that is locally Gorenstein and such that its canonical sheaf verifies $\omega_{X} \cong \mathscr{O}_{X}(-1)$.

As examples of del Pezzo surfaces, we can consider any integral cubic surface in $\mathbb{P}^{3}$ or any complete intersection of two quadrics in $\mathbb{P}^{4}$. Notice that there exists a more general definition of del Pezzo surface for which it is only required that $\omega_{X}^{-1}$ is ample. Smooth surfaces with ample anticanonical sheaf are classically classified; see, for example, [Manin 1986, Chapter IV, Theorems 24.3 and 24.4] or [Dolgachev 2010, Corollary 8.1.17].

Any del Pezzo surface $X \subseteq \mathbb{P}^{d}$ satisfies $\operatorname{deg}(X)=d=\operatorname{codim}(X)+2$. Recall that given a nondegenerate projective variety $X \subseteq \mathbb{P}^{d}$ it always holds that $\Delta(X):=$ $\operatorname{deg} X+\operatorname{dim} X-\mathrm{h}^{0}\left(\mathcal{O}_{X}(1)\right) \geq 0$. It is a classical result the classification of minimal varieties, i.e., varieties for which there is equality in the previous expression (see, for instance, [Dolgachev 2010, Theorem 8.1.1]). Moreover, in the setting of his theory of $\Delta$-genus, Fujita has also a satisfactory classification of quasiminimal varieties, i.e., varieties $X$ satisfying $\Delta(X)=1$. In his terminology, del Pezzo surfaces correspond to quasiminimal surfaces with sectional genus one (i.e., the arithmetic genus of a general hyperplane section is one). For more details see [Fujita 1990]. 
Any del Pezzo surface is ACM [Fujita 1990, (6.4)]. Therefore, according to [Hoa 1993, Theorem 1], the minimal free resolution of the coordinate ring of a del Pezzo surface $X \subseteq \mathbb{P}^{d}$ has the form:

$$
0 \rightarrow R(-d) \rightarrow R(-d+2)^{\alpha_{d-3}} \rightarrow \cdots \rightarrow R(-2)^{\alpha_{1}} \rightarrow R \rightarrow R_{X} \rightarrow 0
$$

where

$$
\alpha_{i}=i\left(\begin{array}{c}
d-1 \\
i+1
\end{array}\right)-\left(\begin{array}{c}
d-2 \\
i-1
\end{array}\right) \text { for } 1 \leq i \leq d-3 .
$$

Notice that $X$ turns out to be AG and, in particular, $\alpha_{i}=\alpha_{d-2-i}$ for all $i=$ $1, \ldots, d-2$.

\section{The minimal resolution conjecture for sets of $m(r)$ and $n(r)$ general points}

The goal of this section is to prove MRC for general sets of points of two specific cardinalities

$$
m(r):=\frac{1}{2} d r^{2}+\frac{1}{2} r(2-d) \quad \text { and } \quad n(r):=\frac{1}{2} d r^{2}+\frac{1}{2} r(d-2)
$$

on a del Pezzo surface $X$. Since the structure of our proof requires that $X$ contains at least a line $L$ and moreover that the elements of the linear system $|L+r H|$ satisfy condition $G_{1}$ in order to apply the theory of generalized divisors, we need to exclude the four cases $X \cong \mathbb{P}^{2}, X \cong \mathbb{P}^{1} \times \mathbb{P}^{1}, X \cong \mathrm{F}_{2}:=\mathbb{P}\left(\mathcal{O}_{\mathbb{P}^{1}} \oplus \mathcal{O}_{\mathbb{P}^{1}}(-2)\right)$, and $X$ the Bordello surface, a complete intersection of two quadrics on $\mathbb{P}^{4}$ with a double line. Therefore, in this section $X \subseteq \mathbb{P}^{d}$ will stand for any del Pezzo surface as was defined in Definition 2.14 except the four aforementioned sporadic cases. The Hilbert polynomial and the regularity of $X$ can be easily computed using (2-2): $P_{X}(r)=\frac{1}{2} d\left(r^{2}+r\right)+1$ and $\operatorname{reg} X=3$. Notice that

$$
P_{X}(r-1)<m(r)<n(r)<P_{X}(r) .
$$

We also set the following notation.

(i) $L$ is any line on $X$.

(ii) $H$ denotes a general hyperplane section of $X$.

(iii) If $C$ is a curve on $X, H_{C}$ will be a general hyperplane section of $C$ and $K_{C}$ the canonical divisor on $C$.

The strategy for finding the minimal free resolution for a general set of points with cardinality $n(r)$ or $m(r)$, for $r \geq 2$, is as follows. First we establish the result for $m(2)=d+2$ points, which gives the starting point for our induction process. Then, using G-liaison, we prove that if $m(r)$ general points on any del Pezzo surface satisfy MRC then so do $n(r)$ general points. Next we observe that if $n(r)$ general points on $X$ have the expected minimal free resolution then $n(r)+1$ 
general points do as well. And, finally, we show that if $n(r)+1$ general points on a del Pezzo surface satisfy MRC then so do $m(r+1)$.

We will prove the result via a series of lemmas and propositions. Since the shape of the minimal free resolution of the homogeneous ideal $I_{X \mid \mathbb{P}^{3}}$ of a del Pezzo surface of degree 3 is slightly different from that of a del Pezzo surface of degree $d \geq 4$ we need to consider the two cases separately in some arguments. We will give complete proofs in the case of degree $d \geq 4$. The concrete arguments on the case of degree 3 are analogous but much easier to write down and will therefore be left to the reader.

Lemma 3.1. Let $X \subseteq \mathbb{P}^{d}$ be any del Pezzo surface of degree $d \geq 4$ and take $C \in$ $|(r+\epsilon) H|, r \geq 2, \epsilon \in\{0,1\}$. Then, any effective divisor $G$ in the linear system $\left|r H_{C}\right|$ is $A G$ and it has a minimal free resolution of the following form:

$$
\begin{array}{r}
0 \rightarrow R(-2 r-d-\epsilon) \rightarrow R(-2 r-d+2-\epsilon)^{\alpha_{d-3}} \oplus R(-r-d)^{2-\epsilon} \oplus R(-r-d-1)^{\epsilon} \\
\rightarrow \cdots \rightarrow M_{i} \rightarrow R(-2 r-\epsilon) \oplus R(-r-2)^{(2-\epsilon) \alpha_{1}} \oplus R(-r-3)^{\epsilon \alpha_{1}} \\
\rightarrow M_{1}:=R(-r)^{2-\epsilon} \oplus R(-r-1)^{\epsilon} \rightarrow I_{G \mid X} \rightarrow 0,
\end{array}
$$

where $M_{i}:=R(-2 r-i+1-\epsilon)^{\alpha_{i-2}} \oplus R(-r-i)^{(2-\epsilon) \alpha_{i-1}} \oplus R(-r-i-1)^{\epsilon \alpha_{i-1}}$ for $i=3, \ldots, d-2$ and $\alpha_{i}=i\left(\begin{array}{c}d-1 \\ i+1\end{array}\right)-\left(\begin{array}{c}d-2 \\ i-1\end{array}\right)$ for $1 \leq i \leq d-3$.

Proof. A curve $C$ in $|(r+\epsilon) H|$ has saturated ideal $I_{C \mid X}=\mathrm{H}_{*}^{0}\left(\mathrm{O}_{X}(-r-\epsilon)\right)$. From (2-2) we have

$$
0 \rightarrow \mathrm{O}_{\mathbb{P} d}(-d) \rightarrow \mathbb{O}_{\mathbb{p} d}(-d+2)^{\alpha_{d-3}} \rightarrow \cdots \rightarrow \mathrm{O}_{\mathbb{p} d}(-2)^{\alpha_{1}} \rightarrow \mathrm{O}_{\mathbb{p} d} \rightarrow \mathrm{O}_{X} \rightarrow 0,
$$

with $\alpha_{i}=i\left(\begin{array}{c}d-1 \\ i+1\end{array}\right)-\left(\begin{array}{c}d-2 \\ i-1\end{array}\right)$ for $1 \leq i \leq d-3$. Twisting (3-2) with $\bigcirc_{\mathbb{p} d}(-r-\epsilon)$ and taking global sections we get the minimal graded free resolution of $I_{C \mid X}$ :

$$
\begin{aligned}
0 \rightarrow R(-r-d-\epsilon) \rightarrow \cdots \rightarrow & R(-r-(i+\epsilon))^{\alpha_{i-1}} \\
& \rightarrow \cdots \rightarrow R(-r-2-\epsilon)^{\alpha_{1}} \rightarrow R(-r-\epsilon) \rightarrow I_{C \mid X} \rightarrow 0 .
\end{aligned}
$$

Now we can apply the horseshoe lemma to the exact sequence

$$
0 \rightarrow I_{X \mid \mathbb{P} d} \rightarrow I_{C \mid \mathbb{P} d} \rightarrow I_{C \mid X} \rightarrow 0
$$

to obtain the minimal free resolution of $I_{C \mid \mathbb{P} d}$ :

$$
\begin{aligned}
0 \rightarrow R(-r-d-\epsilon) \rightarrow & R(-r-d+2-\epsilon)^{\alpha_{d-3}} \oplus R(-d) \rightarrow \cdots \\
& \rightarrow T_{i}:=R(-r-i-\epsilon)^{\alpha_{i-1}} \oplus R(-(i+1))^{\alpha_{i}} \rightarrow \cdots \\
& \rightarrow R(-r-\epsilon) \oplus R(-2)^{\alpha_{1}} \rightarrow I_{C \mid \mathbb{P} d} \rightarrow 0 .
\end{aligned}
$$

This sequence shows that $C \subseteq \mathbb{P}^{d}$ is an arithmetically Gorenstein variety with canonical module $K_{C}:=E x t_{R}^{d-1}\left(R / I_{C}, R(-d-1)\right)=R_{C}(r-1+\epsilon)$. Therefore 
$I_{G \mid C}=H_{*}^{0}\left(\mathcal{O}_{C}(-r)\right)=K_{C}(-2 r+1-\epsilon)$. We apply $\operatorname{Hom}(-, R(-d-1))$ to the previous sequence and we get a graded minimal free resolution of $K_{C}$ :

$0 \rightarrow R(-d-1) \rightarrow R(r-d-1+\epsilon) \oplus R(-d+1)^{\alpha_{d-3}} \rightarrow \cdots$

$$
\rightarrow T_{i}^{\prime} \rightarrow \cdots \rightarrow R(-1) \oplus R(r-3+\epsilon)^{\alpha_{1}} \rightarrow R(r-1+\epsilon) \rightarrow K_{C} \rightarrow 0,
$$

where $T_{i}^{\prime}:=T_{d-i}^{\vee}(-d-1)=R(r-i-\epsilon)^{\alpha_{i-1}} \oplus R(-i)^{\alpha_{i-2}}$ for $i=3, \ldots, d-2$. If we twist the previous sequence by $-2 r+1-\epsilon$ we get the minimal resolution of $I_{G \mid C}$ :

$$
\begin{aligned}
0 \rightarrow R(-2 r-d-\epsilon) \rightarrow & R(-r-d) \oplus R(-2 r-d+2-\epsilon)^{\alpha_{d-3}} \rightarrow \cdots \rightarrow T_{i}^{\prime}(-2 r+1-\epsilon) \\
& \rightarrow \cdots \rightarrow R(-2 r-\epsilon) \oplus R(-r-2)^{\alpha_{1}} \rightarrow R(-r) \rightarrow I_{G \mid C} \rightarrow 0 .
\end{aligned}
$$

Finally, we can apply the horseshoe lemma to the short exact sequence

$$
0 \rightarrow I_{C \mid X} \rightarrow I_{G \mid X} \rightarrow I_{G \mid C} \rightarrow 0
$$

to recover the resolution of $I_{G \mid X}$ :

$$
\begin{array}{r}
0 \rightarrow R(-2 r-d-\epsilon) \rightarrow R(-2 r-d+2-\epsilon)^{\alpha_{1}} \oplus R(-r-d)^{2-\epsilon} \oplus R(-r-d-1)^{\epsilon} \\
\rightarrow \cdots \rightarrow M_{i} \rightarrow \cdots \rightarrow R(-2 r-\epsilon) \oplus R(-r-2)^{(2-\epsilon) \alpha_{1}} \oplus R(-r-3)^{\epsilon \alpha_{1}} \\
\rightarrow R(-r)^{2-\epsilon} \oplus R(-r-1)^{\epsilon} \rightarrow I_{G \mid X} \rightarrow 0
\end{array}
$$

where $M_{i}:=R(-2 r-i+1-\epsilon)^{\alpha_{i-2}} \oplus R(-r-i)^{(2-\epsilon) \alpha_{i-1}} \oplus R(-r-i-1)^{\epsilon \alpha_{i-1}}$ for $i=3, \ldots, d-2$.

Lemma 3.2. Let $X \subseteq \mathbb{P}^{3}$ be a del Pezzo surface of degree 3 and take $C \in|(r+\epsilon) H|$, $r \geq 2, \epsilon \in\{0,1\}$. Then, any effective divisor $G$ in the linear system $\left|r H_{C}\right|$ is $A G$ and it has a minimal free resolution of the form

$$
\begin{aligned}
0 \rightarrow R(-2 r-3-\epsilon) \rightarrow R(-2 r-\epsilon) \oplus R(-r-3)^{2-\epsilon} \oplus R(-r-4)^{\epsilon} & \\
& \rightarrow R(-r)^{2-\epsilon} \oplus R(-r-1)^{\epsilon} \rightarrow I_{G \mid X} \rightarrow 0 .
\end{aligned}
$$

Proof. This is completely analogous to Lemma 3.1. See also [Casanellas 2009, Proposition 3.5].

Lemma 3.3. Let $X \subseteq \mathbb{P}^{d}$ be a del Pezzo surface and let $L \subseteq X$ be a line on it. Take $C \in|L+r H|, r \geq 2$, and let $G$ be any effective divisor in the linear system $\left|2 r H_{C}-K_{C}\right|$. Then, $G$ is arithmetically Gorenstein and the minimal free resolution of $I_{G \mid C}$ has the form

$$
\begin{aligned}
0 \rightarrow & R(-2 r-d-1) \rightarrow R(-2 r-d+1)^{\alpha_{1}} \oplus R(-r-d)^{d-1} \rightarrow \cdots \\
& \rightarrow R(-2 r-i)^{\alpha_{d-i}} \oplus R(-r-i-1)^{\left(\begin{array}{c}
d-1 \\
d-i
\end{array}\right)+\alpha_{d-i-1}} \rightarrow \cdots \\
& \rightarrow R(-2 r-1) \oplus R(-r-3)^{\left(\begin{array}{l}
d-1 \\
d-2
\end{array}\right)+\alpha_{d-3}} \rightarrow R(-r-1) \oplus R(-r-2) \rightarrow I_{G \mid C} \rightarrow 0,
\end{aligned}
$$


with $\alpha_{i}=i\left(\begin{array}{c}d-1 \\ i+1\end{array}\right)-\left(\begin{array}{c}d-2 \\ i-1\end{array}\right)$ for $1 \leq i \leq d-3$.

Proof. Let $L \subseteq X$ be any line. Its ideal as a subvariety of $\mathbb{P}^{d}$ has a resolution

$$
0 \rightarrow R(-d+1) \rightarrow \cdots \rightarrow R(-i)^{\left(\begin{array}{c}
d-1 \\
i
\end{array}\right)} \rightarrow \cdots \rightarrow R(-1)^{d-1} \rightarrow I_{L \mid \mathbb{P}^{d}} \rightarrow 0 .
$$

Using the mapping cone procedure for the exact sequence $0 \rightarrow I_{X \mid \mathbb{P}^{d}} \rightarrow I_{L \mid \mathbb{P}^{d}} \rightarrow$ $I_{L \mid X} \rightarrow 0$ we get

$0 \rightarrow R(-d) \oplus R(-d+1) \rightarrow \cdots \rightarrow R(-i)^{\left(\begin{array}{c}d-1 \\ i\end{array}\right)+\alpha_{i-1}} \rightarrow \cdots \rightarrow R(-1)^{d-1} \rightarrow I_{L \mid X} \rightarrow 0$

with $\alpha_{i}=i\left(\begin{array}{c}d-1 \\ i+1\end{array}\right)-\left(\begin{array}{c}d-2 \\ i-1\end{array}\right)$ for $1 \leq i \leq d-3$. Therefore, $C \in|L+r H|$ has the minimal graded free resolution

$$
\begin{aligned}
0 \rightarrow R(-r-d) \oplus R(-r-d+1) \rightarrow & \cdots \rightarrow R(-r-i)^{\left(\begin{array}{c}
d-1 \\
i
\end{array}\right)+\alpha_{i-1}} \\
& \rightarrow \cdots \rightarrow R(-r-1)^{d-1} \rightarrow I_{C \mid X} \rightarrow 0 .
\end{aligned}
$$

Now the horseshoe lemma applied to $0 \rightarrow I_{X \mid \mathbb{P}^{d}} \rightarrow I_{C \mid \mathbb{P}^{d}} \rightarrow I_{C \mid X} \rightarrow 0$ gives us $0 \rightarrow R(-r-d) \oplus R(-r-d+1) \rightarrow R(-r-d+2)^{\left(\begin{array}{l}d-1 \\ d-2\end{array}\right)+\alpha_{d-3}} \oplus R(-d)$

$$
\begin{aligned}
\rightarrow \cdots \rightarrow R(-r-i) & \left(\begin{array}{c}
d-1 \\
i
\end{array}\right)+\alpha_{i-1} \oplus R(-(i+1))^{\alpha_{i}} \rightarrow \cdots \rightarrow \\
R(-r-1)^{d-1} \oplus R(-2)^{\alpha_{1}} & \rightarrow I_{C \mid \mathbb{P} d} \rightarrow 0 .
\end{aligned}
$$

Since $C$ is $A C M$ we can apply $\operatorname{Hom}(-, R(-d-1))$ to get a resolution of $K_{C}$ :

$$
\begin{aligned}
0 \rightarrow R(-d-1) \rightarrow & R(-d+1)^{\alpha_{1}} \oplus R(r-d)^{d-1} \\
\rightarrow & \cdots \rightarrow R(r-i-1)^{\left(\begin{array}{l}
d-1 \\
d-i
\end{array}\right)+\alpha_{d-i-1}} \oplus R(-i)^{\alpha_{d-i}} \rightarrow \cdots \rightarrow \\
& \quad R(r-3)^{\left(\begin{array}{l}
d-1 \\
d-2
\end{array}\right)+\alpha_{d-3}} \oplus R(-1) \rightarrow R(r-1) \oplus R(r-2) \rightarrow K_{C} \rightarrow 0 .
\end{aligned}
$$

Now, since $G \in\left|2 r H_{C}-K_{C}\right|$ we have

$$
\begin{aligned}
0 \rightarrow & R(-2 r-d-1) \rightarrow R(-2 r-d+1)^{\alpha_{1}} \oplus R(-r-d)^{d-1} \\
& \rightarrow \cdots \rightarrow R(-2 r-i)^{\alpha_{d-i}} \oplus R(-r-i-1)^{\left(\begin{array}{c}
d-1 \\
d-i
\end{array}\right)+\alpha_{d-i-1}} \rightarrow \cdots \rightarrow \\
& \quad R(-2 r-1) \oplus R(-r-3)^{\left(\begin{array}{l}
d-1 \\
d-2
\end{array}\right)+\alpha_{d-3}} \rightarrow R(-r-1) \oplus R(-r-2) \rightarrow I_{G \mid C} \rightarrow 0 .
\end{aligned}
$$

Lemma 3.4. Let $X \subseteq \mathbb{P}^{3}$ be an integral cubic surface and let $L \subseteq X$ be a line on it. Take $C \in|L+r H|, r \geq 2$, and let $G$ be any effective divisor in the linear system $\left|2 r H_{C}-K_{C}\right|$. Then, $G$ is arithmetically Gorenstein and the minimal free resolution of $I_{G \mid C}$ has the following form:

$0 \rightarrow R(-2 r-4) \rightarrow R(-2 r-1) \oplus R(-r-3)^{2} \rightarrow R(-r-1) \oplus R(-r-2) \rightarrow I_{G \mid C} \rightarrow 0$

Proof. This is completely analogous to Lemma 3.3. 
Lemma 3.5. A general set $Z$ of $m(2)=d+2$ points on any del Pezzo surface $X \subseteq \mathbb{P}^{d}$ has a minimal free resolution of the type

$$
0 \rightarrow R(-d-2) \rightarrow R(-d)^{\gamma_{d-1}} \rightarrow \cdots \rightarrow R(-3)^{\gamma_{2}} \rightarrow R(-2)^{2 d-1} \rightarrow I_{Z \mid X} \rightarrow 0,
$$

with

$$
\gamma_{i}=\sum_{l=0}^{1}(-1)^{l}\left(\begin{array}{c}
d-l-1 \\
i-l
\end{array}\right) \Delta^{l+1} H_{X}(2+l)-\left(\begin{array}{c}
d \\
i
\end{array}\right)\left(m(2)-H_{X}(1)\right) .
$$

Proof. A general set $Z$ of $d+2$ points on $X$ is in linearly general position (i.e., any subset of $Z$ of $d+1$ points spans $\left.\mathbb{P}^{d}\right)$. It is well-known that such a $Z$ is AG with minimal free resolution

$$
\begin{aligned}
0 \rightarrow R(-d-2) \rightarrow R(-d)^{\rho_{d-1}} \rightarrow & R(-d+1)^{\rho_{d-2}} \\
& \rightarrow \cdots \rightarrow R(-3)^{\rho_{2}} \rightarrow R(-2)^{\rho_{1}} \rightarrow I_{Z \mid \mathbb{P}^{d}} \rightarrow 0,
\end{aligned}
$$

where $\rho_{i}=i\left(\begin{array}{c}d+1 \\ i+1\end{array}\right)-\left(\begin{array}{c}d \\ i-1\end{array}\right)$ for $1 \leq i \leq d-1$. We now apply the mapping cone procedure to $0 \rightarrow I_{X} \rightarrow I_{Z} \rightarrow I_{Z \mid X} \rightarrow 0$ to obtain a free resolution of $I_{Z \mid X}$ :

$0 \rightarrow R(-d-2) \rightarrow R(-d)^{\rho_{d-1}+1} \rightarrow R(-d+1)^{\rho_{d-2}} \rightarrow R(-d+2)^{\rho_{d-3}-\alpha_{d-3}}$

$$
\rightarrow \cdots \rightarrow R(-3)^{\rho_{2}-\alpha_{2}} \rightarrow R(-2)^{\rho_{1}-\alpha_{1}} \rightarrow I_{Z \mid X} \rightarrow 0,
$$

with $\alpha_{i}=i\left(\begin{array}{c}d-1 \\ i+1\end{array}\right)-\left(\begin{array}{c}d-2 \\ i-1\end{array}\right)$ for $1 \leq i \leq d-3$. Since there are no ghost terms on the previous exact sequence, it is minimal and the coefficients are forced to be given by the formula from the statement.

Once we have fixed the starting point of the induction we can deal with the different steps of the procedure.

Fix an integer $r \geq 2$ and let $Z_{m(r)}$ and $Z_{n(r)}$ be general sets of points on $X$ of cardinality $m(r)$ and $n(r)$ respectively. We show that they are directly $G$-linked by an effective divisor $G$ linearly equivalent to $r H_{C}$, where $C$ is a curve in the linear system $\left|r H_{X}\right|$. Two issues need to be checked. First, we must show that $\mathrm{h}^{0}\left(\mathrm{O}_{X}(r)\right)>m(r)$, to guarantee the existence of a curve $C$ in the linear system $\left|r H_{X}\right|$ such that $Z_{m(r)}$ lies on $C$. Secondly, we need to verify that $n(r)>p_{a}(C)$, to be able to apply Riemann-Roch Theorem for (singular) curves, which assures that there exists an effective divisor $Z_{n(r)}$ of degree $n(r)$ such that $Z_{m(r)}+Z_{n(r)}$ is linearly equivalent to a divisor $r H_{C}$. Notice that, thanks to [Eisenbud 2005, Chapter IV, Theorem 4.2], $P_{X}(r)=H_{X}(r)=\mathrm{h}^{0}\left(\mathcal{O}_{X}(r)\right)$ for any $r \geq 1$.

Regarding the first issue, we have $\mathrm{h}^{0}\left(\mathrm{O}_{X}(r)\right)=P_{X}(r)>m(r)$ by construction and by (3-1).

Regarding the second issue, consider the exact sequence

$$
0 \rightarrow \mathrm{O}_{X}(-r) \rightarrow \mathrm{O}_{X} \rightarrow \mathrm{O}_{C} \rightarrow 0 .
$$


Applying the functor of global sections we have

$$
0=H^{1}\left(X, \mathscr{O}_{X}\right) \rightarrow H^{1}\left(C, \mathscr{O}_{C}\right) \rightarrow H^{2}\left(X, \mathscr{O}_{X}(-r)\right) \rightarrow H^{2}\left(X, \mathscr{O}_{X}\right)=0
$$

and therefore $p_{a}(C)=\mathrm{h}^{1}\left(\mathscr{O}_{C}\right)=\mathrm{h}^{2}\left(\mathcal{O}_{X}(-r)\right)=\mathrm{h}^{0}\left(\mathscr{O}_{X}(r-1)\right)$, where the last equality holds by Serre duality and taking into account that $\omega_{X} \cong \mathfrak{O}_{X}(-1)$. Then, since

$$
n(r)=d r^{2}-m(r)>P_{X}(r-1)=\mathrm{h}^{0}\left(\mathrm{O}_{X}(r-1)\right)=p_{a}(C),
$$

we are done.

Since this construction can also be performed starting from a general set $Z_{n(r)}$ of $n(r)$ points we see that a general set of $m(r)$ points is $G$-linked to a general set of $n(r)$ points and vice versa. This yields:

Proposition 3.6. Fix $r \geq 2$ and assume that the ideal $I_{Z_{m(r)} \mid X}$ of $m(r)$ general points on a del Pezzo surface $X \subseteq \mathbb{P}^{d}$ has the minimal free resolution

$$
\begin{aligned}
0 \rightarrow R(-r-d)^{r-1} \rightarrow & R(-r-d+2)^{\gamma_{d-1, r-1}} \\
& \rightarrow \cdots \rightarrow R(-r-1)^{\gamma_{2, r-1}} \rightarrow R(-r)^{(d-1) r+1} \rightarrow I_{Z_{m(r)} \mid X} \rightarrow 0,
\end{aligned}
$$

with

$$
\gamma_{i, r-1}=\sum_{l=0}^{1}(-1)^{l}\left(\begin{array}{c}
d-l-1 \\
i-l
\end{array}\right) \Delta^{l+1} P_{X}(r+l)-\left(\begin{array}{c}
d \\
i
\end{array}\right)\left(m(r)-P_{X}(r-1)\right) .
$$

Then the ideal $I_{Z_{n(r)} \mid X}$ of $n(r)$ general points has the minimal free resolution

$$
\begin{aligned}
0 \rightarrow R(-r-d)^{(d-1) r-1} \rightarrow & R(-r-d+1)^{\beta_{d-1, r}} \\
& \rightarrow \cdots \rightarrow R(-r-2)^{\beta_{2, r}} \rightarrow R(-r)^{r+1} \rightarrow I_{Z_{n(r)} \mid X} \rightarrow 0,
\end{aligned}
$$

with

$$
\beta_{i, r}=\sum_{l=0}^{1}(-1)^{l+1}\left(\begin{array}{c}
d-l-1 \\
i-l
\end{array}\right) \Delta^{l+1} P_{X}(r+l)+\left(\begin{array}{l}
d \\
i
\end{array}\right)\left(n(r)-P_{X}(r-1)\right) .
$$

Conversely, if $n(r)$ general points on a del Pezzo surface $X \subseteq \mathbb{P}^{d}$ have the expected resolution then $m(r)$ general points do as well.

Proof. As mentioned before, we give the complete proof in the case $d \geq 4$. The case $d=3$ is completely analogous using Lemma 3.2 instead of Lemma 3.1. So suppose that $d \geq 4$. We will check that if $m(r)$ general points have the expected resolution then so do $n(r)$ and we leave to the reader the converse (which is proved analogously). By the preceding discussion, $m(r)$ and $n(r)$ general points on $X$ are G-linked by $G \in\left|r H_{C}\right|$, where $C$ is a curve in the linear system $|r H|$. Thanks to Lemma 3.1 we know the resolution of $I_{G \mid X}$ and hence we can apply the mapping 
cone procedure to the commutative diagram

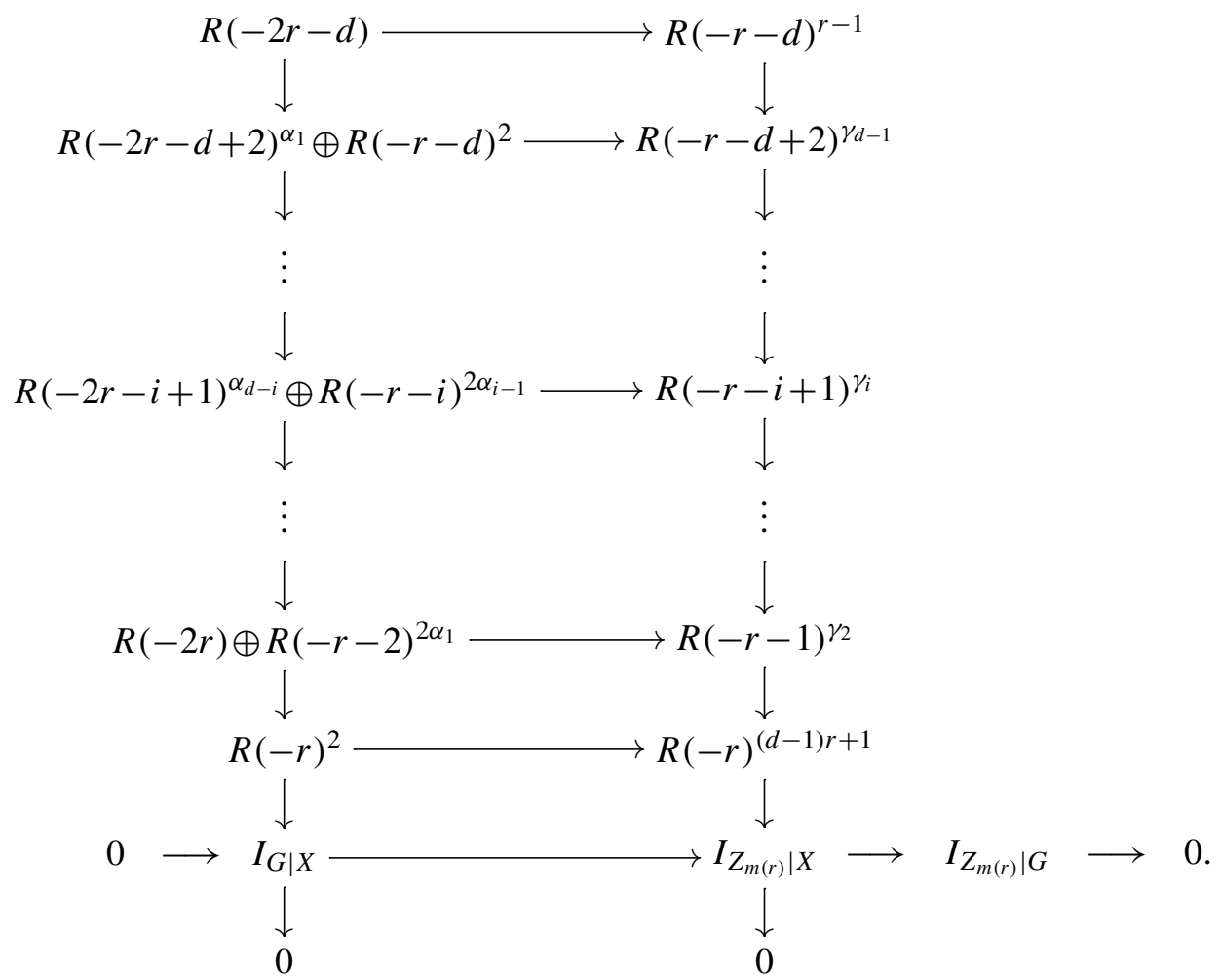

Since $I_{G \mid X} \subseteq I_{Z_{m(r)} \mid X}$, we can take as part of the generators of $I_{Z_{m(r)} \mid X}$ the generators of $I_{G \mid X}$ and therefore the matrix defining the first horizontal map contains nonzero scalar entries. So the repeated elements can be split off. Therefore we get $0 \rightarrow R(-r-d)^{(d-1) r-1} \rightarrow R(-r-d+1)^{\beta_{d-1, r}}$

$$
\rightarrow \cdots \rightarrow R(-2)^{\alpha_{1}} \oplus R(-r)^{r+1} \rightarrow I_{Z_{n(r)} \mid \mathbb{P}^{d}} \rightarrow 0 .
$$

The mapping cone procedure applied to the exact sequence $0 \rightarrow I_{X} \rightarrow I_{Z_{n(r)}} \rightarrow$ $I_{Z_{n(r)} \mid X} \rightarrow 0$ then gives the desired minimal resolution for $I_{Z_{n(r)} \mid X}$.

Lemma 3.7. Let $X \subset \mathbb{P}^{d}$ be any del Pezzo surface. Fix $r \geq 2$ and assume that the ideal $I_{Z_{n(r)} \mid X}$ of a set $Z_{n(r)}$ of $n(r)$ general points on $X \subseteq \mathbb{P}^{d}$ has the expected minimal free graded resolution then a set of $n(r)+1$ general points do as well.

Proof. Since $I_{Z_{n(r)} \mid X}$ has the expected minimal free resolution, we know that $I_{Z_{n(r)} \mid X}$ is generated by $r+1$ forms of degree $r$. Moreover, we know that there are no linear relations among them. We take a general point $p \in X$ and set $Z:=Z_{n(r)} \cup\{p\}$. Since $I_{Z \mid X} \subset I_{Z_{n(r)} \mid X}$, we can take the $r$ generators of $I_{Z \mid X}$ in degree $r$ to be a subset of the generators of $I_{Z_{n(r)} \mid X}$ in degree $r$; in particular, they do not have linear syzygies. 
We must add $d$ generators of degree $r+1$ in order to get a minimal system of generators of $I_{Z \mid X}$. Hence the first module in the minimal free resolution of $I_{Z \mid X}$ is $R(-r)^{r} \oplus R(-r-1)^{d}$ which forces the remaining part of the resolution.

Proposition 3.8. Let $X \subseteq \mathbb{P}^{d}$ be a del Pezzo surface. Fix $r \geq 2$ and assume that the ideal $I_{Z_{p(r)} \mid X}$ of $p(r):=n(r)+1$ general points on $X$ has the minimal free resolution

$$
\begin{aligned}
0 \rightarrow R(-r-d)^{(d-1) r} & \rightarrow R(-r-d+1)^{\delta_{d-1, r}} \\
\rightarrow \cdots & \rightarrow R(-r-2)^{\delta_{2, r}} \rightarrow R(-r)^{r} \oplus R(-r-1)^{d} \rightarrow I_{Z_{p(r)} \mid X} \rightarrow 0,
\end{aligned}
$$

with

$$
\delta_{i, r}=\sum_{l=0}^{1}(-1)^{l+1}\left(\begin{array}{c}
d-l-1 \\
i-l
\end{array}\right) \Delta^{l+1} H_{X}(r+l)+\left(\begin{array}{l}
d \\
i
\end{array}\right)\left(p(r)-H_{X}(r-1)\right) .
$$

Then the ideal $I_{Z_{m(r+1)} \mid X}$ of $m(r+1)$ general points has the minimal free resolution $0 \rightarrow R(-r-d-1)^{r} \rightarrow R(-r-d+1)^{\gamma_{d-1, r}}$

$$
\rightarrow \cdots \rightarrow R(-r-2)^{\gamma_{2}, r} \rightarrow R(-r-1)^{(d-1) r+d} \rightarrow I_{Z_{m(r+1)} \mid X} \rightarrow 0,
$$

with

$$
\gamma_{i, r}=\sum_{l=0}^{1}(-1)^{l}\left(\begin{array}{c}
d-l-1 \\
i-l
\end{array}\right) \Delta^{l+1} H_{X}(r+1+l)-\left(\begin{array}{c}
d \\
i
\end{array}\right)\left(m(r+1)-H_{X}(r)\right) .
$$

Proof. Let $Z_{p(r)}$ be a set of $p(r)$ general points with resolution as in the statement. Let us consider the linear system $|L+r H|$. Since $\operatorname{dim}|L+r H| \geq \operatorname{dim}|r H|=$ $h^{0}\left(\mathcal{O}_{X}(r)\right)-1=P_{X}(r)-1>p(r)$, we can find a curve $C \in|L+r H|$ passing through these $p(r)$ points. As it is shown in [Pons-Liopis 2011, Chapter II] we can suppose that $C$ verifies condition $G_{1}$. Notice that $\operatorname{deg}(C)=1+r d$ and $p_{a}(C)=$ $d\left(\begin{array}{c}r \\ 2\end{array}\right)+r$. Since $p_{a}(C)<m(r+1)$ we can find an effective divisor $Z_{m(r+1)}$ of degree $m(r+1)$ such that $Z_{p(r)}$ and $Z_{m(r+1)}$ are $G$-linked by a divisor of degree $p(r)+m(r+1)=d r^{2}+d r+2=\operatorname{deg}\left(2 r H_{C}-K_{C}\right)$. This will allowed us to find the resolution of $I_{m(r+1) \mid X}$. First of all, let us find the minimal free resolution of the ideal $I_{p(r) \mid C}$ from the exact sequence $0 \rightarrow I_{C \mid X} \rightarrow I_{p(r) \mid X} \rightarrow I_{p(r) \mid C} \rightarrow 0$ through the mapping cone procedure, with the resolution of $I_{C \mid X}$ as it was found in (3-3). It turns out to be

$$
\begin{aligned}
0 \rightarrow R(-r-d)^{(d-1) r+1} & \rightarrow R(-r-d+1)^{c_{d-1, r}} \\
\rightarrow \cdots & \rightarrow R(-r-2)^{c_{2, r}} \rightarrow R(-r)^{r} \oplus R(-r-1) \rightarrow I_{p(r) \mid C} \rightarrow 0 .
\end{aligned}
$$

Since we have already found out the minimal free resolution of $I_{G \mid C}$ (Lemma 3.3) we can use the mapping cone procedure applied to the sequence $0 \rightarrow I_{G \mid C} \rightarrow$ $I_{p(r) \mid C} \rightarrow I_{p(r) \mid G} \rightarrow 0$ to get 


$$
\begin{aligned}
& 0 \rightarrow R(-2 r-d-1) \rightarrow R(-r-d)^{(d-1) r+d} \oplus R(-2 r-d+1)^{\alpha_{1}} \\
& \rightarrow \cdots \rightarrow R(-r-i)^{d_{i, r}} \oplus R(-2 r-i+1)^{\alpha_{d-i+1}} \rightarrow \cdots \rightarrow \\
& R(-r-2)^{d_{2, r}} \rightarrow R(-r)^{r} \rightarrow I_{Z_{p(r)} \mid G} \rightarrow 0 .
\end{aligned}
$$

(If $d=3$ we have instead $0 \rightarrow R(-2 r-4) \rightarrow R(-r-3)^{2 r+2} \oplus R(-2 r-1) \rightarrow$ $R(-r-2)^{d_{2, r}} \rightarrow R(-r)^{r} \rightarrow I_{Z_{p(r)} \mid G} \rightarrow 0$.)

Finally we obtain the minimal free resolution of $I_{m(r+1) \mid \mathbb{P}^{d}}$ :

$$
\begin{aligned}
& 0 \rightarrow R(-r-d-1)^{r} \rightarrow R(-r-d+1)^{\gamma_{d-1, r}} \rightarrow R(-r-d+2)^{\gamma_{d-2, r}} \oplus R(-d) \\
& \rightarrow \cdots \rightarrow R(-r-i)^{\gamma_{i, r}} \oplus R(-i)^{\alpha_{i}} \rightarrow \cdots \rightarrow \\
& \quad R(-r-1)^{(d-1) r+d} \oplus R(-2)^{\alpha_{1}} \rightarrow I_{Z_{m(r+1)} \mid \mathbb{P} d} \rightarrow 0
\end{aligned}
$$

$\left(0 \rightarrow R(-r-4)^{r} \rightarrow R(-r-2)^{\gamma_{2, r}} \rightarrow R(-r-1)^{2 r+3} \oplus R(-3) \rightarrow I_{Z_{m(r+1)} \mid \mathbb{P}^{3}} \rightarrow 0\right.$ if $d=3$ ) from which it is straightforward to recover the predicted resolution of $I_{Z_{m(r+1) \mid X}}$.

We are now ready to prove the main theorem of this section:

Theorem 3.9. Let $X \subseteq \mathbb{P}^{d}$ be a del Pezzo surface. We have:

(1) Let $Z_{n(r)} \subseteq X$ be a general set of $n(r)$ points, $r \geq 2$. Then the minimal graded free resolution of $I_{Z_{n(r)} \mid X}$ has the form

$$
\begin{aligned}
0 \rightarrow R(-r-d)^{(d-1) r-1} & \rightarrow R(-r-d+1)^{\beta_{d-1, r}} \rightarrow R(-r-d+2)^{\beta_{d-2, r}} \\
& \rightarrow \cdots \rightarrow R(-r-2)^{\beta_{2, r}} \rightarrow R(-r)^{r+1} \rightarrow I_{Z_{n(r)} \mid X} \rightarrow 0,
\end{aligned}
$$

with

$$
\beta_{i, r}=\sum_{l=0}^{1}(-1)^{l+1}\left(\begin{array}{c}
n-l-1 \\
i-l
\end{array}\right) \Delta^{l+1} H_{X}(r+l)+\left(\begin{array}{c}
n \\
i
\end{array}\right)\left(n(r)-H_{X}(r-1)\right) .
$$

(2) Let $Z_{m(r)} \subseteq X$ be a general set of $m(r)$ points, $r \geq 2$. Then its minimal graded free resolution has the form

$$
\begin{aligned}
0 \rightarrow R(-r-d)^{r-1} & \rightarrow R(-r-d+2)^{\gamma_{d-1, r-1}} \\
\rightarrow & \cdots \rightarrow R(-r-1)^{\gamma_{2, r-1}} \rightarrow R(-r)^{(d-1) r+1} \rightarrow I_{Z_{m(r)} \mid X} \rightarrow 0,
\end{aligned}
$$

with

$$
\gamma_{i, r-1}=\sum_{l=0}^{1}(-1)^{l}\left(\begin{array}{c}
n-l-1 \\
i-l
\end{array}\right) \Delta^{l+1} P_{X}(r+l)-\left(\begin{array}{c}
n \\
i
\end{array}\right)\left(m(r)-P_{X}(r-1)\right) .
$$

In particular, Mustață's conjecture works for $n(r)$ and $m(r), r \geq 4$, general points on a del Pezzo surface $X \subseteq \mathbb{P}^{d}$. 
Proof. Lemma 3.5 establishes the result for a set of $m$ (2) general points, the starting point of our induction process. Therefore, the result about the resolution of $I_{Z_{n(r)} \mid X}$ and $I_{Z_{m(r)} \mid X}$ follows using Lemma 3.7, Propositions 3.6 and 3.8 and applying induction.

\section{Main theorem}

In this last section, we are going to prove that MRC holds for a general set of points $Z$ on a del Pezzo surface (excluding three of the four sporadic cases pointed out at the beginning of the previous section: $X \cong \mathbb{P}^{1} \times \mathbb{P}^{1}, X \cong \mathrm{F}_{2}:=\mathbb{P}\left(\mathscr{O}_{\mathbb{P}^{1}} \oplus \mathcal{O}_{\mathbb{P}^{1}}(-2)\right)$, and $X$ the Bordello surface) when the cardinality of $Z$ falls in intervals of the form $\left[P_{X}(r-1), m(r)\right]$ or $\left[n(r), P_{X}(r)\right], r \geq 4$. So for the rest of the paper $X$ will denote any del Pezzo surface excluding these three particular surfaces. We will use the fact that we already know that $n(r)$ and $m(r)$ general points on a del Pezzo surface satisfy MRC together with the following lemma which controls how the bottom lines of the Betti diagram of a set of general points on a projective variety change when we add another general point. This lemma will turn out to be a cornerstone in our proof of MRC for del Pezzo surfaces:

Lemma 4.1. Let $X \subseteq \mathbb{P}^{n}$ be a projective variety with $\operatorname{dim} X \geq 2, \operatorname{reg} X=m$ and with Hilbert polynomial $P_{X}$. Let $s$ be an integer with $P_{X}(r-1) \leq s<P_{X}(r)$ for some $r \geq m+1$, let $Z$ be a set of s general points on $X$ and let $P \in X \backslash Z$ be a general point. We have

(i) $b_{i, r-1}(Z) \geq b_{i, r-1}(Z \cup P)$ for every $i$.

(ii) $b_{i, r}(Z) \leq b_{i, r}(Z \cup P)$ for every $i$.

Proof. See [Mustaţă 1998, Proposition 1.7.].

We are now ready to state the main result of this paper:

Theorem 4.2. Let $X \subseteq \mathbb{P}^{d}$ be a del Pezzo surface. Let $r$ satisfy $r \geq \operatorname{reg} X+1=4$. Then for a general set of points $Z$ on $X$ such that $P_{X}(r-1) \leq|Z| \leq m(r)$ or $n(r) \leq|Z| \leq P_{X}(r)$ the minimal resolution conjecture is true.

Proof. First of all we want to point out that the result was already known in the cases $|Z|=P_{X}(r-1)$ and $|Z|=P_{X}(r)-1$ [Mustaţă 1998, Examples 1 and 2].

On the other hand, the results about Ulrich bundles proved in [Pons-Liopis 2011, Chapter II] and Serre's correspondence allows us to deal with the case of $X \cong \mathbb{P}^{2}$. So let $X$ be any other del Pezzo surface. Let $Z^{\prime}$ be a general set of points of cardinality $\left|Z^{\prime}\right|=n(r)$ and add general points to $Z^{\prime}$ in order to get a set of points $Z$ of cardinality $n(r) \leq|Z| \leq P_{X}(r)$. By Theorem 3.9 we have that $b_{i, r-1}\left(Z^{\prime}\right)=0$ for all $i=2, \ldots, d$. Therefore we can apply Lemma 4.1 to deduce that $b_{i, r-1}(Z)=0$ for all $i=2, \ldots, d$. Thus, by semicontinuity, MRC holds for a general set of $|Z|$ points. 
Now if $|Z| \leq m(r)$, we can add general points to $Z$ in order to have a general set $Z^{\prime}$ including $Z$ and such that $\left|Z^{\prime}\right|=m(r)$. Again from the previous Theorem we have that $b_{i, r}\left(Z^{\prime}\right)=0$ for all $i=1, \ldots, d-1$. So we can use again Lemma 4.1 to deduce that $b_{i, r}(Z)=0$ for all $i=1, \ldots, d-1$ and therefore MRC holds for $Z$.

As a consequence of Theorem 3.9 we will prove that the number of generators of the ideal of a general set of points on a del Pezzo surface is as small as possible and so it is the number of generators of its canonical module as well. In fact, we have:

Theorem 4.3. Let $X \subseteq \mathbb{P}^{d}$ be a del Pezzo surface. Then for a general set of points $Z$ on $X$ such that $|Z| \geq P_{X}(3)$ the Cohen-Macaulay type conjecture and the ideal generation conjecture are true.

Proof. Let $Z$ be a general set of points on our del Pezzo surface $X$. If it is the case that $n(r) \leq|Z| \leq m(r+1)$ the result has been proved on the previous theorem. So we can assume that $m(r)<|Z|<n(r)$ for some $r$. We know that MRC holds for a general set $\left|Z^{\prime}\right|$ of $n(r)$ points on $X, Z \subseteq Z^{\prime}$ and in particular $b_{1, r}\left(Z^{\prime}\right)=0$. Applying Lemma 4.1 inductively we see that $b_{1, r}(Z)=0$. Analogously, since MRC holds for a general set $Z^{\prime \prime}$ of $m(r)$ points, $b_{d, r-1}\left(Z^{\prime \prime}\right)=0$ with $Z^{\prime \prime} \subseteq Z$. Applying once again the same lemma we see that $b_{d, r-1}(Z)=0$.

In the particular case of the cubic surface, since the minimal free resolution of its points has length three, we recover one of the main results of [Miró-Roig and Pons-Llopis 2012] (see also [Migliore and Patnott 2011; Casanellas 2009]):

Theorem 4.4. Let $X \subseteq \mathbb{P}^{3}$ be an integral cubic surface (i.e., a del Pezzo surface of degree three). Then the minimal resolution conjecture holds for a general set of points on $X$ of cardinality $\geq P_{X}(3)=19$.

Proof. By Theorem 4.3 we know that any set $Z$ of general points on $X$ verify the Cohen-Macaulay type conjecture and the ideal generation conjecture. But since the codimension is three there is no further term on the resolution left to consider so the general MRC also holds.

\section{Acknowledgement}

Short before we finished writing this paper we were informed that Migliore and Patnott were able to prove the minimal resolution conjecture for sets of general distinct points of any cardinality on a cubic surface $X \subseteq \mathbb{P}^{3}$ given that $X$ is smooth or it has at most isolated double points [Migliore and Patnott 2011]. We are very grateful to J. Migliore for sending their paper to us, and for the helpful discussions we have had. 


\section{References}

[Ballico and Geramita 1986] E. Ballico and A. V. Geramita, "The minimal free resolution of the ideal of $s$ general points in $\mathbf{P}^{3}$ ", pp. 1-10 in Proceedings of the 1984 Vancouver conference in algebraic geometry (Vancouver, 1984), edited by J. Carrell et al., CMS Conf. Proc. 6, Amer. Math. Soc., Providence, RI, 1986. MR 87j:14079 Zbl 0621.14003

[Casanellas 2009] M. Casanellas, "The minimal resolution conjecture for points on the cubic surface”, Canad. J. Math. 61:1 (2009), 29-49. MR 2009j:13013 Zbl 1171.13009

[Dolgachev 2010] I. Dolgachev, "Classical algebraic geometry: a modern view", lecture notes, 2010, Available at http://www.math.lsa.umich.edu/ idolga/CAG.pdf.

[Eisenbud 2005] D. Eisenbud, The geometry of syzygies: A second course in commutative algebra and algebraic geometry, Graduate Texts in Mathematics 229, Springer, New York, 2005. MR 2005h:13021 Zbl 1066.14001

[Eisenbud et al. 2002] D. Eisenbud, S. Popescu, F.-O. Schreyer, and C. Walter, "Exterior algebra methods for the minimal resolution conjecture", Duke Math. J. 112:2 (2002), 379-395. MR 2003: 13019 Zbl 1035.13008

[Fujita 1990] T. Fujita, Classification theories of polarized varieties, London Mathematical Society Lecture Note Series 155, Cambridge University Press, 1990. MR 93e:14009 Zbl 0743.14004

[Gaeta 1951] F. Gaeta, "Sur la distribution des degrés des formes appartenant à la matrice de l'idéal homogène attaché à un groupe de $N$ points génériques du plan", C. R. Acad. Sci. Paris 233 (1951), 912-913. In French. MR 13,524d Zbl 0043.36104

[Giuffrida et al. 1996] S. Giuffrida, R. Maggioni, and A. Ragusa, "Resolutions of generic points lying on a smooth quadric", Manuscripta Math. 91:4 (1996), 421-444. MR 98d:13011 Zbl 0873. 14041

[Hartshorne 1994] R. Hartshorne, "Generalized divisors on Gorenstein schemes", K-Theory 8:3 (1994), 287-339. MR 95k:14008 Zbl 0826.14005

[Hartshorne 2007] R. Hartshorne, “Generalized divisors and biliaison”, Illinois J. Math. 51:1 (2007), 83-98. MR 2008j:14010 Zbl 1133.14005

[Hirschowitz and Simpson 1996] A. Hirschowitz and C. Simpson, "La résolution minimale de l'idéal d'un arrangement général d'un grand nombre de points dans $\mathbf{P}^{n}$ ", Invent. Math. 126:3 (1996), 467503. MR 97i:13015 Zbl 0877.14035

[Hoa 1993] L. T. Hoa, "On minimal free resolutions of projective varieties of degree $=$ codimension +2”, J. Pure Appl. Algebra 87:3 (1993), 241-250. MR 94e:13024 Zbl 0803.13007

[Kleppe et al. 2001] J. O. Kleppe, J. C. Migliore, R. Miró-Roig, U. Nagel, and C. Peterson, Gorenstein liaison, complete intersection liaison invariants and unobstructedness, Memoirs of the AMS 732, Amer. Math. Soc., Providence, 2001. MR 2002e:14083

[Lorenzini 1993] A. Lorenzini, "The minimal resolution conjecture”, J. Algebra 156:1 (1993), 5-35. MR 94g:13005 Zbl 0811.13008

[Manin 1986] Y. I. Manin, Cubic forms, 2nd ed., North-Holland Mathematical Library 4, NorthHolland Publishing Co., Amsterdam, 1986. MR 87d:11037 Zbl 0582.14010

[Migliore and Patnott 2011] J. Migliore and M. Patnott, "Minimal free resolutions of general points lying on cubic surfaces in $\mathbb{P}^{3}$ ", J. Pure Appl. Algebra 215:7 (2011), 1737-1746. MR 2012f:13029 Zbl 1217.13005

[Miró-Roig and Pons-Llopis 2012] R. M. Miró-Roig and J. Pons-Llopis, "Minimal free resolution for points on surfaces", J. Algebra 357 (2012), 304-318. 
[Mustață 1998] M. Mustață, "Graded Betti numbers of general finite subsets of points on projective varieties”, Matematiche (Catania) 53:3 (1998), 53-81. MR 2000j:13026 Zbl 0943.13010

[Pons-Liopis 2011] J. Pons-Liopis, Ulrich bundles and varieties of wild representation type, Ph.D. thesis, University of Barcelona, 2011.

[Walter 1995] C. H. Walter, "The minimal free resolution of the homogeneous ideal of $s$ general points in $\mathbf{P}^{4}$ ", Math. Z. 219:2 (1995), 231-234. MR 96f:13024 Zbl 0826.14037

[Weibel 1994] C. A. Weibel, An introduction to homological algebra, Cambridge Studies in Advanced Mathematics 38, Cambridge University Press, 1994. MR 95f:18001 Zbl 0797.18001

Communicated by Craig Huneke

Received 2010-07-02 Revised 2011-01-20 Accepted 2011-02-19

miro@ub.edu

Facultat de Matemàtiques,

Department d'Algebra i Geometria, University of Barcelona, Gran Via des les Corts Catalanes 585, 08007 Barcelona, Spain

jfpons@ub.edu

Facultat de Matemàtiques,

Department d'Algebra i Geometria, University of Barcelona, Gran Via des les Corts Catalanes 585, 08007 Barcelona, Spain 


\section{Algebra \& Number Theory}

msp.berkeley.edu/ant

\section{EDITORS}

MANAGING EDITOR

Bjorn Poonen

Massachusetts Institute of Technology

Cambridge, USA

\author{
EDITORIAL BOARD CHAIR \\ David Eisenbud \\ University of California \\ Berkeley, USA
}

\section{BOARD OF EDITORS}

Georgia Benkart

Dave Benson

Richard E. Borcherds

John H. Coates

J-L. Colliot-Thélène

Brian D. Conrad

Hélène Esnault

Hubert Flenner

Edward Frenkel

Andrew Granville

Joseph Gubeladze

Ehud Hrushovski

Craig Huneke

Mikhail Kapranov

Yujiro Kawamata

János Kollár

Yuri Manin

Barry Mazur

Philippe Michel

Susan Montgomery
University of Wisconsin, Madison, USA

University of Aberdeen, Scotland

University of California, Berkeley, USA

University of Cambridge, UK

CNRS, Université Paris-Sud, France

University of Michigan, USA

Universität Duisburg-Essen, Germany

Ruhr-Universität, Germany

University of California, Berkeley, USA

Université de Montréal, Canada

San Francisco State University, USA

Hebrew University, Israel

University of Kansas, USA

Yale University, USA

University of Tokyo, Japan

Princeton University, USA

Northwestern University, USA

Harvard University, USA

École Polytechnique Fédérale de Lausanne

University of Southern California, USA
Shigefumi Mori

Raman Parimala

Jonathan Pila

Victor Reiner

Karl Rubin

Peter Sarnak

Joseph H. Silverman

Michael Singer

Ronald Solomon

Vasudevan Srinivas

J. Toby Stafford

Bernd Sturmfels

Richard Taylor

Ravi Vakil

Michel van den Bergh

Marie-France Vignéras

Kei-Ichi Watanabe

Andrei Zelevinsky

Efim Zelmanov
RIMS, Kyoto University, Japan

Emory University, USA

University of Oxford, UK

University of Minnesota, USA

University of California, Irvine, USA

Princeton University, USA

Brown University, USA

North Carolina State University, USA

Ohio State University, USA

Tata Inst. of Fund. Research, India

University of Michigan, USA

University of California, Berkeley, USA

Harvard University, USA

Stanford University, USA

Hasselt University, Belgium

Université Paris VII, France

Nihon University, Japan

Northeastern University, USA

University of California, San Diego, USA

\section{PRODUCTION}

contact@msp.org

Silvio Levy, Scientific Editor

See inside back cover or www.jant.org for submission instructions.

The subscription price for 2012 is US \$175/year for the electronic version, and \$275/year (+\$40 shipping outside the US) for print and electronic. Subscriptions, requests for back issues from the last three years and changes of subscribers address should be sent to Mathematical Sciences Publishers, Department of Mathematics, University of California, Berkeley, CA 94720-3840, USA.

Algebra \& Number Theory (ISSN 1937-0652) at Mathematical Sciences Publishers, Department of Mathematics, University of California, Berkeley, CA 94720-3840 is published continuously online. Periodical rate postage paid at Berkeley, CA 94704, and additional mailing offices.

ANT peer review and production are managed by EditFLOW ${ }^{\circledR}$ from Mathematical Sciences Publishers.

PUBLISHED BY

mathematical sciences publishers

http://msp.org/

A NON-PROFIT CORPORATION

Typeset in IAT $_{\mathrm{E}} \mathrm{X}$

Copyright (C2012 by Mathematical Sciences Publishers 


\section{Algebra \& Number Theory}

\section{Volume $6 \quad$ No. $1 \quad 2012$}

The Chevalley-Shephard-Todd theorem for finite linearly reductive group schemes MATTHEW SATRIANO

The minimal resolution conjecture for points on del Pezzo surfaces

ROSA M. MIRÓ-ROIG and JOAN PONS-LLOPIS

$L$-series of Artin stacks over finite fields

SHENGHAO SUN

Multiplicative mimicry and improvements to the Pólya-Vinogradov inequality LEO GOLDMAKHER

Quiver Grassmannians and degenerate flag varieties

Giovanni Cerulli Irelli, Evgeny Feigin and Markus Reineke 